\title{
Genomic imbalances in 70 snap-frozen cervical squamous intraepithelial lesions: associations with lesion grade, state of the HPVI6 E2 gene and clinical outcome
}

\author{
W Alazawi ${ }^{1,2}$, M Pett ${ }^{1,2}$, S Strauss ${ }^{3}$, R Moseley $^{4}$, J Gray ${ }^{3}$, M Stanley ${ }^{2}$ and N Coleman ${ }^{*, 1,2,4}$ \\ 'Medical Research Council Cancer Cell Unit, Hutchison/MRC Research Centre, Hills Road, Cambridge CB2 2XZ, UK; '2Department of Pathology, \\ University of Cambridge, Tennis Court Road, Cambridge CB2 IDQ, UK; ${ }^{3}$ Health Protection Agency, Colindale Avenue, London NW9 5HT, UK; \\ ${ }^{4}$ Department of Histopathology, Addenbrooke's Hospital, Hills Road, Cambridge CB2 2QQ, UK
}

\begin{abstract}
Host genomic abnormalities may determine the natural history of cervical squamous intraepithelial lesions (SILs). We undertook comparative genomic hybridisation analysis of epithelium carefully microdissected from 70 cervical SILs, the largest series to date. In contrast to previous studies, we used frozen sections for optimal DNA quality and examined whether patterns of DNA copy number imbalance $(\mathrm{CNI})$ are characteristic of SIL grade, human papillomavirus (HPV) status and postoperative recurrence. We identified more CNIs in cervical SIL than previously described, with more CNIs per case in high-grade squamous intraepithelial lesion (HG-SIL) than in low-grade squamous intraepithelial lesion (LG-SIL) $(P=0.04)$. While some CNIs were seen at similar frequencies in HG-SIL and LG-SIL, others, including gain on Iq, 3q and I6q, were found frequently in HG-SIL but not in LG-SIL. There were significantly more CNIs per case in HG-SILs showing loss of the HPVI 6 E2 gene (a repressor of viral oncogene transcription) $(P=0.026)$ and in HG-SILs that subsequently recurred $(P=0.04)$. Our data are consistent with sequential acquisition of CNIs in cervical SIL progression. Higher frequency of $\mathrm{CNI}$ in association with $\mathrm{E} 2$ gene loss supports in vitro evidence that high-risk HPV integration is associated with genomic instability. Further investigation of the clinical value of specific host genomic abnormalities in cervical SIL is warranted.
\end{abstract} British Journal of Cancer (2004) 91, 2063-2070. doi:I0.1038/sj.bjc.6602237 www.bjcancer.com

Published online 16 November 2004

(c) 2004 Cancer Research UK

Keywords: $\mathrm{CGH}$; papillomavirus; cervix; recurrence

Cervical squamous cell carcinoma (SCC) usually arises from a subset of high-grade squamous intraepithelial lesions (HG-SIL), which in turn are thought to arise from a subset of low-grade squamous intraepithelial lesions (LG-SIL) (Holowaty et al, 1999; Franco et al, 2001). Untreated SILs have the potential to regress, persist or progress, and up to $8.6 \%$ of SILs recur locally following complete excision (Mohamed-Noor et al, 1997; Hulman et al, 1998; Nagai et al, 2000; Chao et al, 2004). However, the molecular pathology of cervical SILs is poorly understood and it is currently not possible to predict the natural history of an individual lesion. Unnecessary follow-up of women with cervical SILs destined not to progress or recur imposes large burdens on colposcopy services worldwide and produces substantial adverse psychosocial consequences for individual patients. There is therefore a very important requirement for new rational and objective approaches to improving diagnosis and prediction of outcome in cervical SIL.

Infection with high-risk human papillomavirus (HR-HPV) is known to be an independent risk factor for progression of LG-SIL to

*Correspondence: Dr N Coleman, Medical Research Council Cancer Cell Unit, Hutchison/MRC Research Centre, Hills Road, Cambridge CB2 2XZ, UK; E-mail: ncl09@cam.ac.uk

Received 16 August 2004; accepted I October 2004; published online 16 November 2004
HG-SIL and for the development of SCC (Remmink et al, 1995; Konno et al, 1998). However, HPV testing is inherently nonspecific at identifying patients destined to develop cervical SCC (Sasieni, 2000) or HG-SIL (Lorincz et al, 2002). Other biological factors of importance in the progression of SIL or recurrence after treatment include persistence of HR-HPV (Costa et al, 2003), integration of HR-HPV into host chromosomes and the acquisition of secondary host genomic abnormalities (Lazo, 1999). Integration frequently causes disruption of the HR-HPV E2 transcriptional repressor with consequent deregulation of HR-HPV oncogenes, events that we have recently demonstrated to be important in inducing high-level genomic instability in cervical keratinocytes in vitro (Pett et al, 2004).

Very little information is available as to whether particular host genomic abnormalities in cervical SIL may be characteristic of lesion grade, HPV status or clinical outcome. Allelic imbalances have been reported on numerous chromosome arms in cervical carcinomas and small numbers of SILs (Steenbergen et al, 1996; Chu et al, 1999; Lazo, 1999; Pulido et al, 2000), and there is some evidence of progressive accumulation of allelic imbalances with increasing grade of abnormality. (Larson et al, 1997; Chu et al, 1999; Luft et al, 1999; Chung et al, 2000; Lin et al, 2000; Chuaqui et al, 2001).

Likewise, data from comparative genomic hybridisation (CGH) have been reported for only relatively small numbers of cervical 
SILs (Heselmeyer et al, 1996; Kirchhoff et al, 1999, 2001; Umayahara et al, 2002). These studies have generally suggested that relatively few copy number imbalances (CNIs) exist in SIL. However, such data should be interpreted with caution, since all studies used formalin-fixed paraffin-embedded tissue, in which DNA degradation may cause difficulties with $\mathrm{CGH}$, and cells microdissected under histological control do not appear to have been analysed in all studies (Kirchhoff et al, 1999, 2001). Moreover, one study combined cases equivalent to LG-SIL with cases equivalent to HG-SIL (Kirchhoff et al, 2001) and another examined SIL adjacent to SCC (Umayahara et al, 2002), which may not represent typical SIL. Most importantly, no study to date has correlated the number and type of CNIs in cervical SIL with key clinicopathological information such as HPV status and postsurgical outcome.

We have therefore performed CGH using metaphase chromosome targets to investigate the number and frequency of CNIs in a large series of 70 snap-frozen cervical SILs from 70 different patients, in which the abnormal epithelium was microdissected from serial frozen sections under histological control. None of the SILs was accompanied by SCC. We had three objectives when performing this work. Firstly, we aimed to characterise the number and locations of CNIs in cervical HG-SIL and LG-SIL. We analysed the stage of disease progression at which individual abnormalities occurred, in order to identify possible pathways of progression. We reasoned that any abnormalities present at similar frequencies in LG-SIL and HG-SIL might represent early events in the pathogenesis of SIL, while abnormalities present at a higher frequency in HG-SIL than in LG-SIL might reflect those involved in disease progression.

Secondly, we sought to identify any associations between CNI and the state of the HPV16 E2 gene in HPV16-positive HG-SILs. Initially, we performed HPV detection and typing on all cases using nested PCR and reverse line hybridisation. In HG-SILs containing HPV16, the HPV type most frequently associated with cervical SCC (Bosch et al, 1995), we examined associations between CNIs and the presence or absence of the HPV16 E2 and E7 genes, as detected by PCR. We aimed to identify cases in which HPV16 E2 was lost but HPV16 E7 was retained, as these cases would be expected to show derepression of HR-HPV oncogenes. Previous validation studies using Southern blotting had shown that such E2-negative/E7-positive cases contained HPV16 integrants in the absence of HPV16 episomes (Das et al, 1992). The E2/E7 PCR technique was employed, as the microdissected samples did not yield sufficient quantities of genomic DNA (gDNA) and RNA to enable us to use optimal techniques for assessment of HR-HPV integration, such as restriction site PCR (Thorland et al, 2000) or amplification of papillomavirus oncogene transcripts (Wentzensen et al, 2002). The third and final aim of our study was to assess the clinical value of selected host and viral parameters in predicting recurrence of HG-SIL after complete excision by large loop excision of the transformation zone (LLETZ).

\section{MATERIALS AND METHODS}

\section{Tissue}

The experimental work was performed with the permission of the Cambridge Local Research Ethics Committee (Ref: 03/023). The study used gDNA extracted from SIL epithelium carefully microdissected from frozen sections of cervical tissue. The tissue was obtained from LLETZ samples removed by a consultant gynaecologist from 70 different patients undergoing treatment for cervical disease. None of the cases was associated with SCC. The LLETZ samples were placed on ice after removal and study tissue was removed and snap-frozen in liquid nitrogen within 30-60 min. Samples were numbered at the time of recruitment. The histopathological diagnosis in the frozen sections for microdissection was agreed by two consultant histopathologists. Of the 70 cases, 51 were HG-SIL (given the prefix H; Figure 1) and 19 LG-SIL (given the prefix L). The median patient age was 30 years (range 19-63 years). Normal cervical epithelium and stroma were obtained from six hysterectomies performed for non-neoplastic disease unrelated to the cervix.

\section{Identification of disease recurrence}

Cases of recurrence following complete local excision were identified from review of patient records by appropriate medical practitioners. The median follow-up period was 45 months (range 11-65 months), which is appropriate for assessment of local recurrence (Mohamed-Noor et al, 1997; Hulman et al, 1998; Nagai et al, 2000; Chao et al, 2004). Recurrence was defined as a diagnosis of SIL of either grade by histology or of dyskaryosis of any grade by cytology provided the original lesion was reported as completely excised and there had been at least one negative smear and/or biopsy between the original LLETZ and the recurrence. 'Borderline' cytological abnormalities were not regarded as recurrences. All samples were anonymised and researchers were blinded to the available clinical data until the CGH analysis was complete.

\section{Microdissection and DNA extraction}

In all, 10 consecutive $10 \mu \mathrm{m}$ sections were cut from each frozen tissue block. The first and last were stained with haematoxylin and eosin (H\&E) and used as guides for identifying SILs in the intervening unstained sections. The abnormal epithelium was microdissected and collected using sterile scalpels under stereoscopic visualisation in a microdissecting microscope at $\times 15-25$ magnification. Some sections were stained by H\&E following microdissection, in order to assess the accuracy of the procedure. In all cases, at least $80 \%$ of the microdissected tissue was composed of abnormal epithelium. The microdissected tissue was placed in $50 \mu \mathrm{l}$ of $10 \mathrm{~mm}$ Tris/1 mM EDTA (pH 8.0) buffer containing $0.4 \mu \mathrm{g}^{-1}$ Proteinase $\mathrm{K}$ (Sigma, Poole UK) and incubated at $37^{\circ} \mathrm{C}$ overnight. Genomic DNA for HPV typing was extracted from lysates of microdissected cells using guanidinium isothiocyanate/silica as described (Boom et al, 1990).

\section{HPV typing}

PCR for HPV detection was performed as described previously (Strauss et al, 1999) using the L1 consensus degenerate primers MY09 and MY11 for the initial amplification (expected product size $452 \mathrm{bp}$ ) followed by a second-round nested PCR using the GP5- and GP6-positive primers (expected product size $150 \mathrm{bp}$ ).

HPV typing was performed by reverse line hybridisation, as described elsewhere (Jordens et al, 2000), using probes complementary to sequences of the L1 region of HPV types $2,6,11,16,18$, $31,33,35,39,41,42,43,45,50,51,52,53,54,56,58,59,61,62,66$, $67,70,72,81$ and Han 831 . HPV types $16,18,31,33,35,39,45,51$, 52, 56, 58, 59 and 66 were regarded as HR-HPV (Munoz et al, 2003), while the other types were regarded as low-risk HPV.

\section{State of HPV16 E2 gene}

Lysates of microdissected cells from HPV16-positive cases (where available) were used to assess the presence or absence of the HPV16 E2 gene, with reference to the HPV16 E7 gene.

A measure of $5 \mu \mathrm{l}$ of lysate was PCR amplified using the AmpliTaq Gold kit (Applied Bioscience, Foster City, CA, USA):

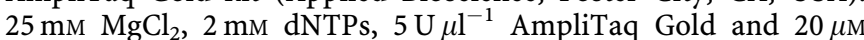
primer pairs for either full-length HPV16 E2 (forward: 5'-TGCGATGGATCCATGGAGACTCTTTGCCAACG-3'; reverse: 


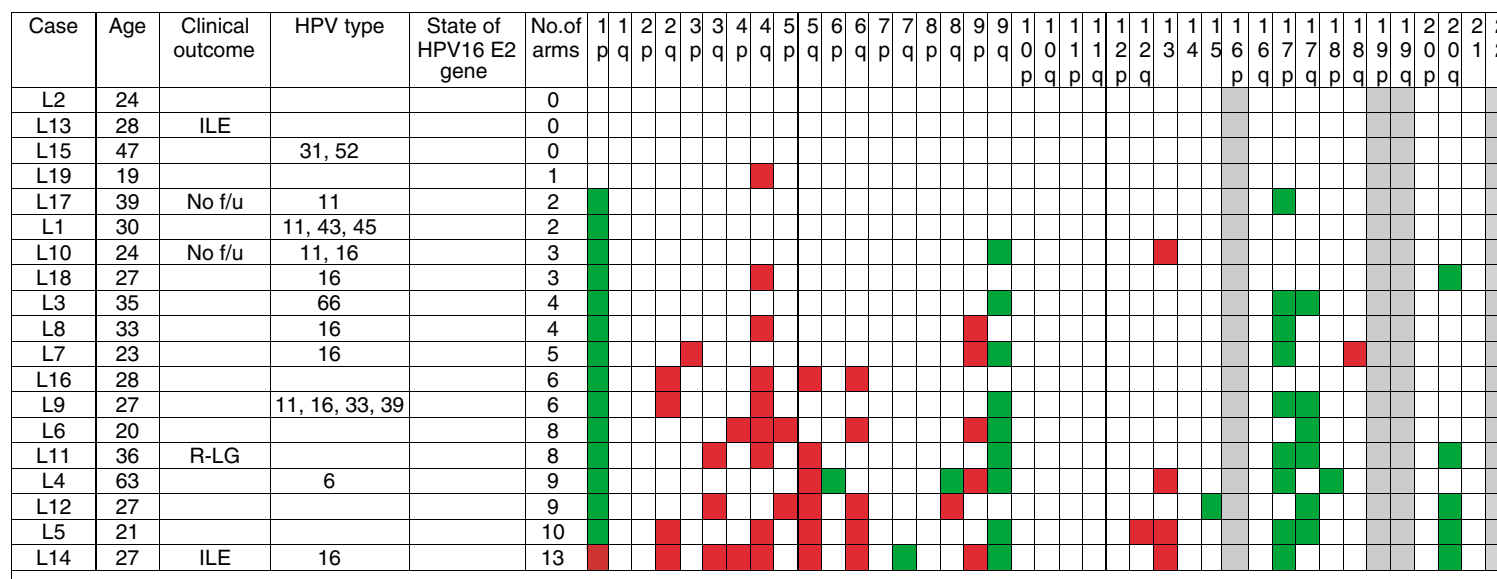

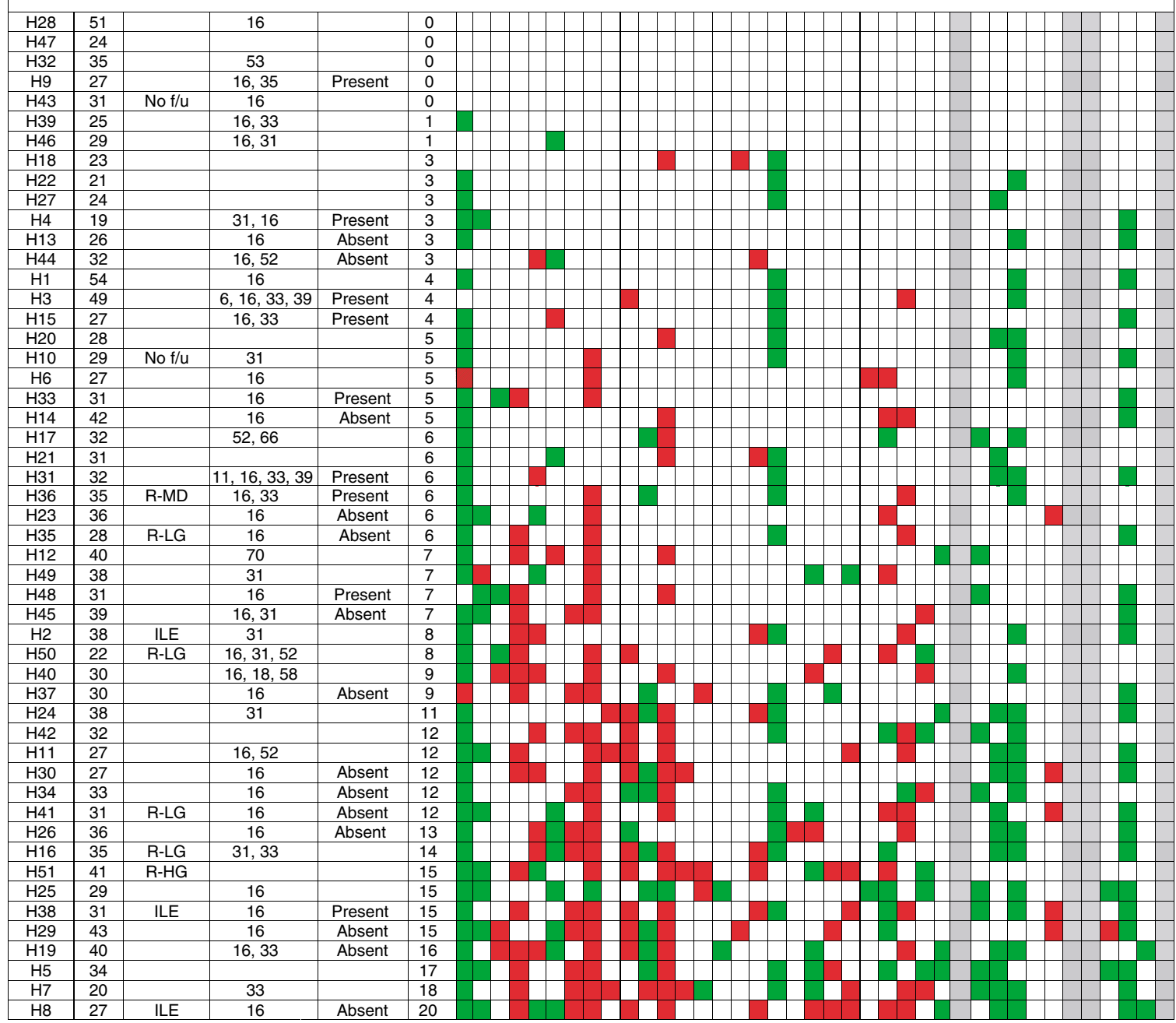

Figure I Clinicopathological data and frequency of CNIs in the 70 SILs studied. Prefix L denotes low-grade SIL and prefix H denotes high-grade SIL. Clinical outcome is shown if noteworthy. (ILE = inadequate local excision; No f/u = no follow-up data available; R-LG= recurred with a histological diagnosis of LG-SIL; R-HG = recurred with a histological diagnosis of HG-SIL; R-MiD = recurred with cytological mild dyskaryosis). Also listed are patient age; HPV types detected in the 53 cases analysed; the presence or absence of the HPVI6 E2 gene in cases of HG-SIL that were HPVI 6 positive and tested ( $n=23$ ); and the number of chromosome arms showing DNA CNI. Cases are sorted by a grade of SIL and then ranked by the number of arms showing CNI. For each arm, white boxes indicate no CNI, green boxes indicate gain, red boxes indicate loss and green/red striped boxes indicate gain and loss on the same arm. No data are shown for chromosomes 16p, 19 and 22, for which analysis by CGH is unreliable. In all 23 cases of HG-SIL tested for the state of the HPVI6 E2 gene, the HPVI6 E7 gene was detectable.

5'-TGCGATGGATTCTCATATAGACATAAATCCAG-3'; expected product size $1139 \mathrm{bp}$ ) or full-length HPV16 E7 (forward $5^{\prime}$ ATGCATGGAGATACACCTAC- ${ }^{\prime}$; reverse $5^{\prime}$-TGGTTTCTGAGAA CAGATGGG-3'; expected product size 294 bp). HPV16 DNA in the
pSPHPV-16 plasmid (Stanley et al, 1989) was used as a positive control.

Samples were considered positive for a particular reaction when a band of the appropriate size was clearly identified. Cases showing 
equivocal positivity were not encountered in this study. All amplifications were preformed in triplicate. On every occasion, the presence or absence of a PCR product band was consistent for all three replicates.

\section{Comparative genomic hybridisation}

A measure of $5 \mu \mathrm{l}$ of lysate of microdissected cells was used in a primary degenerate oligonucleotide primed (DOP) PCR reaction as described previously (Roberts et al, 1999). Test probes were made by labelling $250 \mathrm{ng}$ of primary DOP products in a secondary DOP reaction incorporating digoxigenin-11-dUTP (Boehringer Mannheim, Germany). Reference DNA was obtained from normal male peripheral blood lymphocytes and subjected to two rounds of DOP-PCR, using biotin-16-dUTP in the secondary labelling reaction (Boehringer Mannheim, Germany). The test and reference DNAs were sex mismatched in order to provide an internal control.

Probes were made by ethanol coprecipitating $500 \mathrm{ng}$ of test and reference products together with $5 \mu \mathrm{g}$ of Cot-1 DNA (Roche Diagnostics, Lewes, UK). Hybridisation to normal male metaphase spreads (Vysis, Richmond, UK) was as described previously (Sanoudou et al, 2000; Roberts et al, 2001). The biotin and digoxigenin labels were detected using avidin-Cy3 (Amersham Pharmacia Biotech, Little Chalfont, UK) and antidigoxigenin fluorescein isothiocyanate (FITC)-conjugated Fab fragments (Roche Diagnostics, Lewes, UK), respectively (Roberts et al, 2001).

For each case, seven to 12 metaphases were captured on an Axioplan II epifluorescence microscope (Zeiss, Welwyn Garden City, UK) equipped with narrow bandpass filter blocks for DAPI, Cy3 and FITC, a Sensys charge-coupled device camera (Photometrics, Tucson, AZ, USA) and SmartCapture VP imaging software (Digital Scientific, Cambridge, UK). Images were assessed using Quips CGH Analysis and Interpretation software (Vysis, Richmond, UK). Nine normal-normal control hybridisations were performed using six test samples from microdissected normal ectocervical epithelium and three test samples from microdissected normal cervical stroma. Based on the results of these hybridisations, the green-to-red (test-to-reference) fluorescence intensity ratio thresholds were set to 0.85 for loss and 1.15 for gain.

Chromosomes 16p, 19 and 22 were excluded from the CGH analysis as they yield unreliable CGH data (Kallioniemi et al, 1994; Zitzelsberger et al, 1997). The telomere of chromosome 1p was not excluded, as the SIL samples showed DNA gain at this region rather than DNA loss (which can represent a spurious finding; Zitzelsberger et al, 1997), with no evidence of such gain in the normal-normal hybridisations.

\section{Statistical analysis}

Differences in the number of CNIs per case between sample groups were compared using the Mann-Whitney $U$-test. Differences in the frequency of individual CNIs between sample groups were compared using the $\chi^{2}$ test.

\section{RESULTS}

\section{Clinical follow-up data}

Of the 70 SILs examined by CGH, five had been inadequately excised and follow-up data were not available for four (Figure 1). In total, 61 cases (46 HG-SIL; 15 LG-SIL) were therefore completely excised and accompanied by adequate follow-up data. The median follow-up time was 45 months (range 11-65 months). Seven cases recurred (11\%), of which six were HG-SIL (H16, H35, H36, H41, H50 and H51) and one was LG-SIL (L11). Recurrence was diagnosed by histological examination in all of these cases, except H36 where it was diagnosed by cytology. The median time for recurrence of the HG-SILs was 15 months (range 11-22 months).

\section{HPV typing}

Sufficient DNA was available for HPV testing following CGH in 53 of the 70 cases. HPV DNA was detected in all of these cases. In all, 15 different HPV types were detected and multiple infections were seen in $20(38 \%)$ cases. HR-HPV types were detected in 39 of 42 (93\%) of the testable HG-SILs and in eight of $11(73 \%)$ of the testable LG-SILs (Figure 1). HPV16 was detected in 39 cases (33 HG-SIL, six LG-SIL), but only one case (an HG-SIL) was HPV18 positive.

\section{E2/E7 PCR}

A total of 23 HPV16-positive HG-SILs had sufficient residual DNA to permit PCR assessment of the presence of the HPV16 E2 and E7 genes (Figure 2). In total, 14 cases (61\%) harboured HPV16 E7 but not HPV16 E2, consistent with integrated HPV16 in the absence of HPV16 episomes. Nine cases (39\%) harboured intact HPV16 E2 and HPV16 E7 genes.

\section{Comparative genomic hybridisation}

Associations with lesion grade The summary of CGH copy number karyograms for all 70 cases examined are shown in Figure 3 and the number and locations of chromosome arms involved for each case is shown in Figure 1. There were more CNI per case in HG-SIL (median 6 , range $0-20 ; n=51$ ) than in LG-SIL (median 4 , range $0-13 ; n=19)(P=0.04)$ (Figures 1 and $4 \mathrm{a}$ ).

The most frequently occurring abnormalities in the LG-SILs were gain on $1 \mathrm{p}(79 \%), 9 \mathrm{q}(47 \%), 17 \mathrm{p}(37 \%), 17 \mathrm{q}(32 \%)$ and $20 \mathrm{q}$ $(32 \%)$ and loss on 4q (47\%), 5q (32\%), 2q (26\%), 6q (26\%), 9p $(26 \%)$ and $13 q(21 \%)$. The most frequently occurring abnormalities in the HG-SILs were gain on $1 p(80 \%), 17 q(47 \%), 20 q(47 \%)$, $9 \mathrm{q}(45 \%)$ and $17 \mathrm{p}(27 \%)$ and loss on $4 \mathrm{q}(53 \%), 6 \mathrm{q}(43 \%), 2 \mathrm{q}(33 \%)$, 13q $(25 \%)$ and 5q (24\%).

Certain consistent regions of common gain and loss were identified, particularly gain at 1 pter-1p32, 3q14-21, 6p21.3-21.2 and $9 \mathrm{q} 34$ and loss at $2 \mathrm{q} 22-32,4 \mathrm{q} 22-28,5 \mathrm{q} 14-23,6$ cen-q21, 9q21, $11 q 12-13,11 q 14-21,12 q 15-21$ and $13 q 21-22$. We also noted rarer gains at 3 p21, $14 q 24$ and $15 \mathrm{q} 22$ and rarer losses at $7 \mathrm{p} 21,7 \mathrm{q} 21$ and $14 \mathrm{q} 12-13$. No regions of amplification (test-to-reference fluorescence ratio $>1.5$ ) were seen in any case.

Figure 5 shows the frequency of gain and loss of selected chromosome arms in all cases examined. Some CNIs occurred at essentially similar frequencies in HG-SIL and LG-SIL, including gains on $1 \mathrm{p}$ and $9 \mathrm{q}$ and losses on $4 \mathrm{q}, 5 \mathrm{q}, 6 \mathrm{q}$ and $13 \mathrm{q}$. In contrast, gains on $1 \mathrm{q}, 3 \mathrm{q}$ and $16 \mathrm{q}$ were found frequently in HG-SIL but not

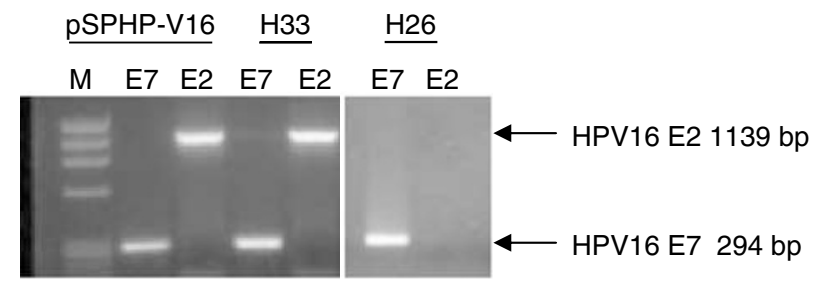

Figure 2 E2/E7 PCR in HPVI6-positive HG-SILs. PCR for HPVI6 E2 and HPVI6 E7 genes in representative cases of HPVI6-positive HG-SILs. M = $\Phi \times$ DNA ladder (HT Biotechnologies Ltd, Cambridge, UK). The positive control pSPHPV-16 plasmid contains full-length HPVI6. Whereas case $\mathrm{H} 33$ contains intact E7 and E2, H26 contains intact E7 but disrupted E2. 

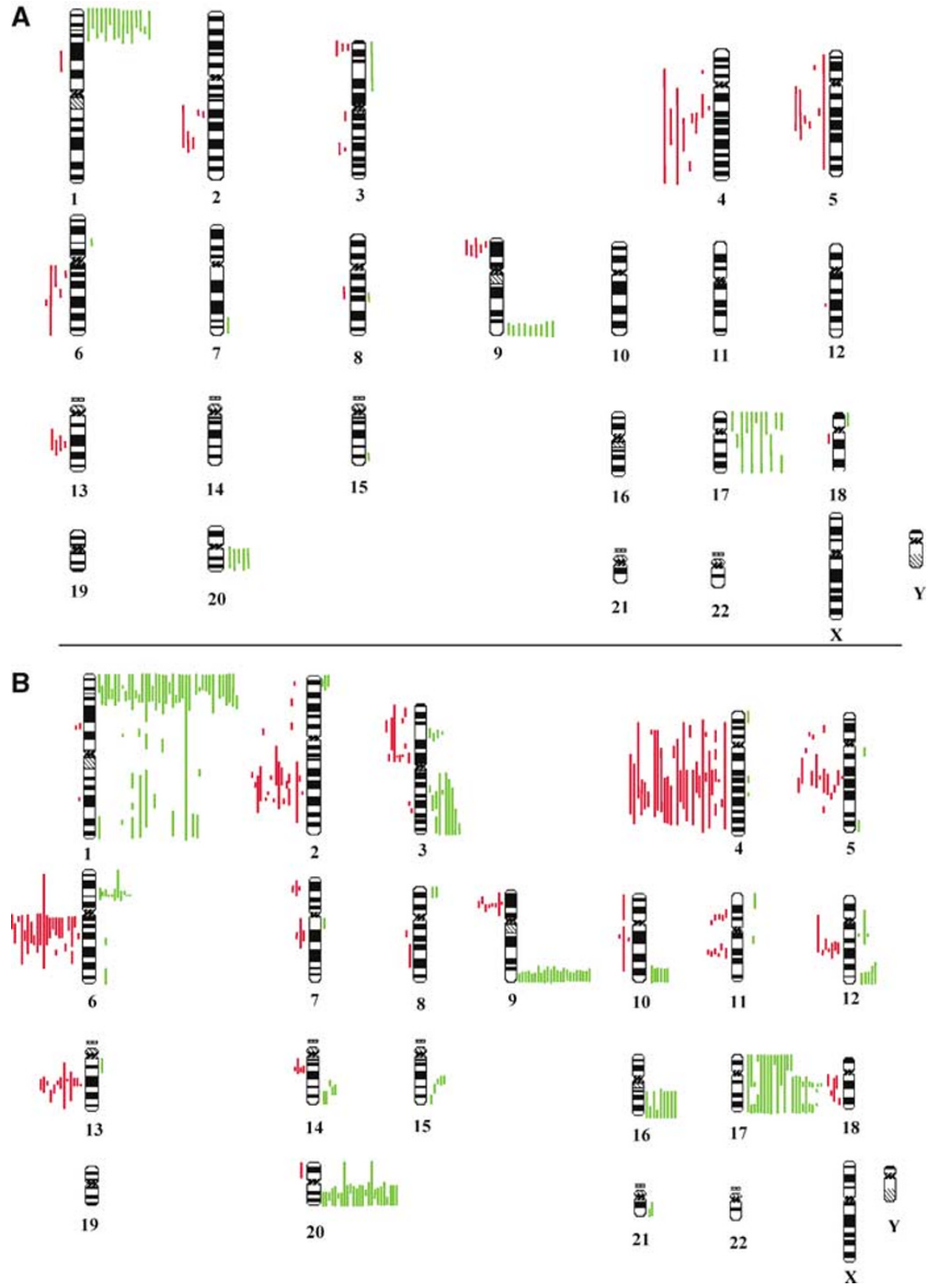

Figure 3 Summary copy number karyogram for 19 LG-SILs $(\mathbf{A})$ and 5 I HG-SILs (B). Each green bar to the right of a chromosome represents a region of DNA gain in a single case and each red bar to the left of a chromosome represents a region of DNA loss in a single case.

at all in LG-SIL $(P<0.05$ for each). Gain on $3 q$ was seen in 10 cases of HG-SIL (20\%) and was the only abnormality in one case. Gain of $6 \mathrm{p}$ was also seen frequently in HG-SIL (22\%) but only in a single LG-SIL.

Associations with state of the HPV16 E2 gene in HPV16-positive HG-SIL There were more CNIs per case in the 14 HPV16-positive HG-SIL cases with loss of the HPV16 E2 gene and retention of the HPV16 E7 gene (median 10.5, range 3-20), compared to the nine HPV16-positive HG-SIL cases with intact HPV16 E2 and HPV16 E7 (median 5, range $0-15)(P=0.026)$ (Figure $4 \mathrm{~b})$. The cases with loss of E2 also showed more frequent gain on $3 \mathrm{q}(P<0.05)$. On the other hand, we did observe high numbers of CNIs in some cases of HG-SIL in which the E2 gene was retained (e.g. case H38, which had 15 aberrant chromosome arms).
Associations with clinical outcome in HG-SIL Of the 46 cases of completely excised HG-SIL with adequate follow-up data, there were more CNIs in the six cases that were destined to recur (median 10, range 6-15) than in the 40 that did not recur (median 5.5 , range $0-18)(P=0.04)$ (Figure $4 \mathrm{c})$. Loss of $4 \mathrm{q}(P=0.01)$ and loss of $5 \mathrm{q}(P<0.05)$ were more frequent in the HG-SILs destined to recur than in those that did not recur.

\section{DISCUSSION}

We have identified more CNIs per case than previously reported in SIL. We observed a median of four CNIs per case in LG-SIL and six CNIs per case in HG-SIL, compared, for example, to a mean of 1.1 CNIs per case in lesions equivalent to LG-SIL and a mean of 4.1 


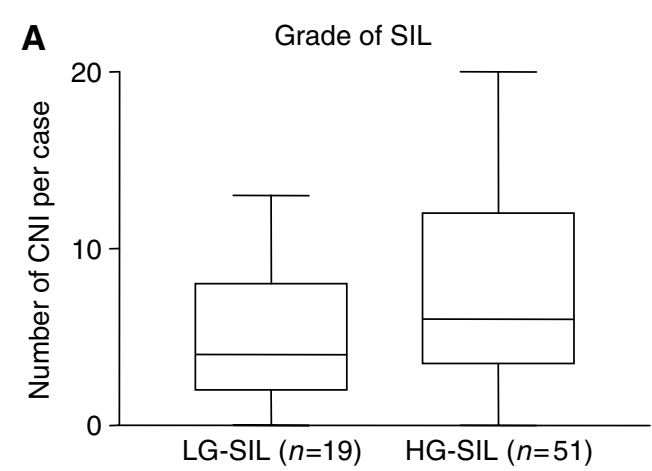

B State of HPV16 E2 gene in HPV16-positive HG-SIL

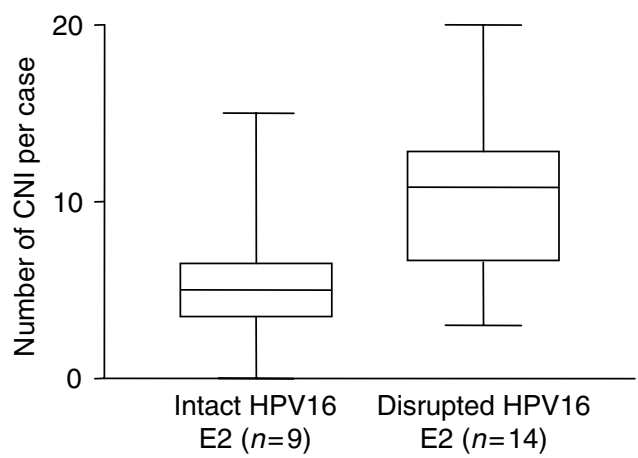

C Outcome post-LLETZ for HG-SIL

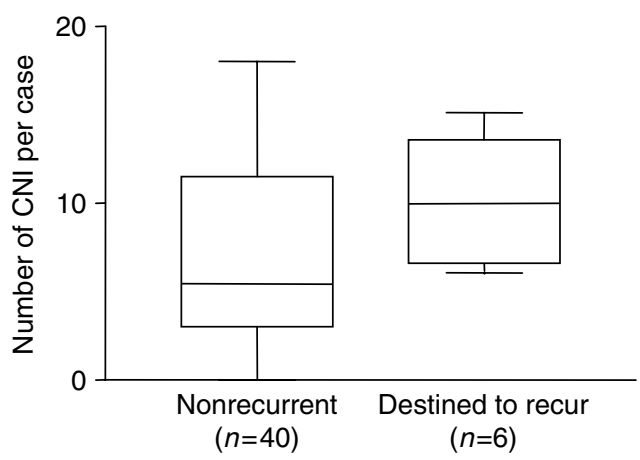

Figure 4 Number of CNIs per case according to selected clinicopathological features. Plots show the median (line), interquartile range (box) and full range (whiskers) of numbers of chromosome arms showing CNI per case in different groups of cases. There are significantly more $\mathrm{CN}$ ls per case in: (A) HG-SILs vs LG-SILs $(P=0.04)$; (B) HPVI6-positive HG-SIL with disrupted HPVI6 E2 vs HPVI6-positive HG-SIL with intact HPVI6 E2 $(P=0.026)$ and $(\mathbf{C})$ HG-SIL destined to recur post-LLETZ vs HG-SIL that did not recur $(P=0.04)$.

CNIs per case in lesions equivalent to HG-SIL in a previous report (Umayahara et al, 2002). Our study has the advantages of using frozen sections of cervical SILs (unaccompanied by SCC) from which the lesional epithelium was microdissected, which may allow for greater sensitivity in detecting CNIs. On the other hand, it should be noted that the thresholds used for determination of copy number gain and loss have been greater in previous studies of cervical SIL (Heselmeyer et al, 1996; Kirchhoff et al, 1999; Umayahara et al, 2002).

Our data indicate that certain recurrent CNIs occur in both cervical LG-SIL and HG-SIL and suggest that there may also be sequential acquisition of imbalances during the progression from LG-SIL to HG-SIL. While the CNIs that occur at similar frequencies

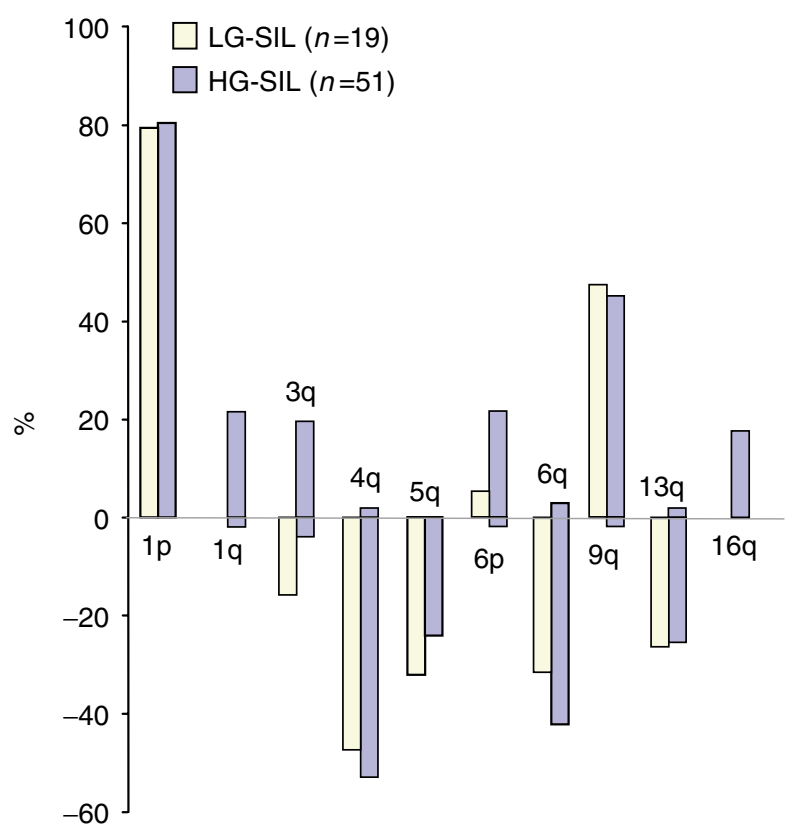

Figure 5 Frequency of selected CNIs in LG- and HG-SILs. Bars above the $x$-axis indicate the percentage frequency of gains on a chromosomal arm, and bars below the $x$-axis indicate the percentage frequency of losses on a chromosomal arm. Yellow bars = LG-SILs; blue bars = HG-SILs.

in LG-SIL and HG-SIL are consistent with early events, those that are significantly more frequent in HG-SIL may confer a selective advantage to LG-SIL cells and contribute to progression via clonal selection. Alternatively, the latter CNIs may represent an advantageous consequence of increased chromosomal instability in HG-SIL (Pett et al, 2004) and be of greater relevance in favouring subsequent progression to SCC.

CGH losses reported here are consistent with sites of allelic imbalance previously reported in cervical SIL and SCC (Lazo, 1999), including 3p12-3p14.1 (Larson et al, 1997; Chu et al, 1999; Chung et al, 2000), 4p16 (Larson et al, 1997), 4q21-q35 (Larson et al, 1997), 6p14-22 (Chatterjee et al, 2001; Steenbergen et al, 2001), 6q22-q27 (Chuaqui et al, 2001), 9p21 (Lin et al, 2000), 11p14-15 and 11q23 (Luft et al, 1999; Pulido et al, 2000). Further investigation of these sites of potential tumour suppressor genes is warranted.

We observed gain of $3 q$ in $20 \%$ of 51 HG-SIL cases. Whereas this imbalance was initially described as defining the transition to SCC in the cervix (Heselmeyer et al, 1996), it was subsequently reported in eight of $37(22 \%)$ paraffin-embedded 'dysplastic' lesions equivalent to HG-SIL (Kirchhoff et al, 1999; Kirchhoff et al, 2001), an observation that is supported by our data. Interestingly, we found that six of the $10 \mathrm{HG}$-SILs in our study that showed gain of $3 q$ also showed loss on $3 p$ or $13 q$, also supporting the previous suggestion of an association between these abnormalities (Kirchhoff et al, 1999). Our findings are consistent with the earlier identification of $3 q 25-q 27$ as the consensus region of gain.

Cases of HPV16-positive HG-SIL in which the E2 gene was disrupted showed significantly more CNIs and significantly more frequent gain on $3 \mathrm{q}$ than cases in which the $\mathrm{E} 2$ gene was intact. Data from the PCR approach that we used to detect E2 and E7 have previously been shown to correlate with the physical state of HPV in clinical specimens, as determined by Southern blotting (Das et al, 1992). Loss of E2 (with retention of E7) is consistent with the presence of integrated HPV16 in the absence of HPV16 episomes. The presence of the HPV16 E2 gene could either be due to episomal HPV16 or to integrated HPV16 in which the gene is 
retained, for example, following concatamerisation of the integrants. Our finding that the E2 gene was disrupted in 14 of 23 (61\%) testable HG-SILs is consistent with previous PCR data (Tonon et al, 2001). Greater chromosomal instability following loss of the E2 gene is likely to be associated with derepression of the HPV16 oncogenes E7 and E6, which can cooperate in the induction of mitotic defects and genomic instability (Duensing et al, 2000; Plug-DeMaggio et al, 2004).

For some cases at least, loss of HR-HPV E2 may therefore be a significant intermediate step in cervical oncogenesis, occurring between infection with HR-HPV and the development of chromosomal instability. Indeed, these data support our recent in vitro observations that integration of HPV16 in cervical keratinocytes, with disruption of the E2 gene as is typically seen in vivo, is closely associated with the development of high-level genomic instability (Pett et al, 2004). On the other hand, our identification in the present study of cases of HG-SIL that retain the HPV16 E2 gene yet show numerous CNIs (e.g. case H38, with 15 aberrant arms) is consistent with the notion that loss of E2 function is not essential for chromosomal instability to develop in cervical keratinocytes. It should be noted, however, that the low cell yield provided by the microdissection approach that we chose to adopt did not enable us to assess levels of E2 gene expression in the samples that we examined. Moreover, because of clonal heterogeneity within SILs, cells containing the E2 gene may have been different to those showing numerous CNIs.

Of the 46 cases of HG-SIL in our study with histologically clear excision margins and adequate follow-up data, six (13\%) recurred. This rate of local recurrence is broadly similar to that described by other authors (Hulman et al, 1998; Nagai et al, 2000). HG-SILs destined to recur showed significantly more CNIs and significantly more frequent loss of $4 \mathrm{q}$ and $5 \mathrm{q}$ than HG-SILs that did not recur. Our observations were made on relatively small numbers of cases, using a whole genome approach that would not be appropriate for

\section{REFERENCES}

Boom R, Sol CJ, Salimans MM, Jansen CL, Wertheim-van Dillen PM, van der Noordaa J (1990) Rapid and simple method for purification of nucleic acids. J Clin Microbiol 28: 495-503

Bosch FX, Manos MM, Munoz N, Sherman M, Jansen AM, Peto J, Schiffman MH, Moreno V, Kurman R, Shah KV (1995) Prevalence of human papillomavirus in cervical cancer: a worldwide perspective. International biological study on cervical cancer (IBSCC) Study Group. J Natl Cancer Inst 87: 796-802

Chao A, Lin CT, Hsueh S, Chou HH, Chang TC, Chen MY, Lai CH (2004) Usefulness of human papillomavirus testing in the follow-up of patients with high-grade cervical intraepithelial neoplasia after conization. Am J Obstet Gynecol 190: 1046-1051

Chatterjee A, Pulido HA, Koul S, Beleno N, Perilla A, Posso H, Manusukhani M, Murty VV (2001) Mapping the sites of putative tumor suppressor genes at $6 \mathrm{p} 25$ and $6 \mathrm{p} 21.3$ in cervical carcinoma: occurrence of allelic deletions in precancerous lesions. Cancer Res 61: 2119-2123

Chu TY, Shen CY, Lee HS, Liu HS (1999) Monoclonality and surface lesionspecific microsatellite alterations in premalignant and malignant neoplasia of uterine cervix: a local field effect of genomic instability and clonal evolution. Genes Chromosomes Cancer 24: 127-134

Chuaqui R, Silva M, Emmert-Buck M (2001) Allelic deletion mapping on chromosome $6 \mathrm{q}$ and $\mathrm{X}$ chromosome inactivation clonality patterns in cervical intraepithelial neoplasia and invasive carcinoma. Gynecol Oncol 80: $364-371$

Chung TK, Cheung TH, Lo WK, Yu MY, Hampton GM, Wong HK, Wong YF (2000) Loss of heterozygosity at the short arm of chromosome 3 in microdissected cervical intraepithelial neoplasia. Cancer Lett 154: $189-194$

Costa S, De Simone P, Venturoli S, Cricca M, Zerbini ML, Musiani M, Terzano P, Santini D, Cristiani P, Syrjanen S, Syrjanen K (2003) Factors predicting human papillomavirus clearance in cervical intraepithelial neoplasia lesions treated by conization. Gynecol Oncol 90: 358-365 routine clinical use. However, our data support the notion that detection of one or more specific host cell abnormalities (either alone or in combination with tests of HPV type, physical state, etc.) may ultimately prove to be of value in risk stratification and clinical management of cervical SILs.

It is likely that shared risk factors underlie the association that we have observed between the number of CNIs in HG-SIL and lesional recurrence despite complete local excision. Most important may be viral factors, such as persistence and integration of HR-HPV (Cuzick, 1997; Cruickshank et al, 2002). However, there may also be a role for host genetic polymorphisms, such as those affecting TP53 (Klug et al, 2001) or local host immunity (Cuzick et al, 2000; Maciag et al, 2000), and/or environmental factors such as smoking, local hormonal effects and other sexually transmitted diseases (Kjellberg et al, 2000).

In summary, we have obtained molecular cytogenetic data that are consistent with a model of sequential acquisition of genomic CNIs in the development and progression of cervical SIL. We show that a high number of CNIs, as well as particular sites of imbalance, are associated with loss of the HPV16 E2 gene in HPV16-positive HG-SIL and with recurrence of HG-SIL after adequate local excision. It may ultimately be possible to improve the prediction of postsurgical outcome in SIL by assessing specific genomic abnormalities in the excised lesion. Our findings now require validation in a different, larger group of cases, using higher resolution approaches.

\section{ACKNOWLEDGEMENTS}

We are most grateful to Mr Ralph Robinson for constructive clinical support. We also thank Lesley Morris, Kate Bird and John Brown for technical assistance.
Cruickshank ME, Sharp L, Chambers G, Smart L, Murray G (2002) Persistent infection with human papillomavirus following the successful treatment of high grade cervical intraepithelial neoplasia. Bjog 109: $579-581$

Cuzick J. (1997) Viral load as a surrogate for persistence in cervical human papillomavirus infection. In New Developments in Cervical Cancer Screening and Prevention Franco E, Monsonego J (eds) Oxford, UK: Blackwell Science

Cuzick J, Terry G, Ho L, Monaghan J, Lopes A, Clarkson P, Duncan I (2000) Association between high-risk HPV types, HLA DRB1* and DQB1* alleles and cervical cancer in British women. Br J Cancer 82: 1348-1352

Das BC, Sharma JK, Gopalakrishna V, Luthra UK (1992) Analysis by polymerase chain reaction of the physical state of human papillomavirus type 16 DNA in cervical preneoplastic and neoplastic lesions. J Gen Virol 73: $2327-2336$

Duensing S, Lee LY, Duensing A, Basile J, Piboonniyom S, Gonzalez S, Crum CP, Munger K (2000) The human papillomavirus type 16 E6 and E7 oncoproteins cooperate to induce mitotic defects and genomic instability by uncoupling centrosome duplication from the cell division cycle. Proc Natl Acad Sci USA 97: $10002-10007$

Franco EL, Duarte-Franco E, Ferenczy A (2001) Cervical cancer: epidemiology, prevention and the role of human papillomavirus infection. Cmaj 164: 1017-1025

Heselmeyer K, Schrock E, du Manoir S, Blegen H, Shah K, Steinbeck R, Auer G, Ried T (1996) Gain of chromosome 3q defines the transition from severe dysplasia to invasive carcinoma of the uterine cervix. Proc Natl Acad Sci USA 93: 479-484

Holowaty P, Miller AB, Rohan T, To T (1999) Natural history of dysplasia of the uterine cervix. J Natl Cancer Inst 91: 252-258

Hulman G, Pickles CJ, Gie CA, Dowling FM, Stocks PJ, Dixon R (1998) Frequency of cervical intraepithelial neoplasia following large loop excision of the transformation zone. J Clin Pathol 51: 375-377 
Jordens JZ, Lanham S, Pickett MA, Amarasekara S, Abeywickrema I, Watt PJ (2000) Amplification with molecular beacon primers and reverse line blotting for the detection and typing of human papillomaviruses. $J$ Virol Methods 89: 29-37

Kallioniemi OP, Kallioniemi A, Piper J, Isola J, Waldman FM, Gray JW, Pinkel D (1994) Optimizing comparative genomic hybridization for analysis of DNA sequence copy number changes in solid tumors. Genes Chromosomes Cancer 10: $231-243$

Kirchhoff M, Rose H, Petersen BL, Maahr J, Gerdes T, Lundsteen C, Bryndorf T, Kryger-Baggesen N, Christensen L, Engelholm SA, Philip J (1999) Comparative genomic hybridization reveals a recurrent pattern of chromosomal aberrations in severe dysplasia/carcinoma in situ of the cervix and in advanced-stage cervical carcinoma. Genes Chromosomes Cancer 24: $144-150$

Kirchhoff M, Rose H, Petersen BL, Maahr J, Gerdes T, Philip J, Lundsteen C (2001) Comparative genomic hybridization reveals non-random chromosomal aberrations in early preinvasive cervical lesions. Cancer Genet Cytogenet 129: $47-51$

Kjellberg L, Hallmans G, Ahren AM, Johansson R, Bergman F, Wadell G, Angstrom T, Dillner J (2000) Smoking, diet, pregnancy and oral contraceptive use as risk factors for cervical intra-epithelial neoplasia in relation to human papillomavirus infection. $\mathrm{Br} J$ Cancer 82: $1332-1338$

Klug SJ, Wilmotte R, Santos C, Almonte M, Herrero R, Guerrero I, Caceres E, Peixoto-Guimaraes D, Lenoir G, Hainaut P, Walboomers JM, Munoz N (2001) TP53 polymorphism, HPV infection, and risk of cervical cancer. Cancer Epidemiol Biomarkers Prev 10: 1009-1012

Konno R, Paez C, Sato S, Yajima A, Fukao A (1998) HPV, histologic grade and age. Risk factors for the progression of cervical intraepithelial neoplasia. J Reprod Med 43: $561-566$

Larson AA, Liao SY, Stanbridge EJ, Cavenee WK, Hampton GM (1997) Genetic alterations accumulate during cervical tumorigenesis and indicate a common origin for multifocal lesions. Cancer Res 57: $4171-4176$

Lazo PA (1999) The molecular genetics of cervical carcinoma. Br J Cancer 80: $2008-2018$

Lin WM, Michalopulos EA, Dhurander N, Cheng PC, Robinson W, Ashfaq R, Coleman RL, Muller CY (2000) Allelic loss and microsatellite alterations of chromosome 3 p14.2 are more frequent in recurrent cervical dysplasias. Clin Cancer Res 6: 1410-1414

Lorincz AT, Castle PE, Sherman ME, Scott DR, Glass AG, Wacholder S, Rush BB, Gravitt PE, Schussler JE, Schiffman M (2002) Viral load of human papillomavirus and risk of CIN3 or cervical cancer. Lancet 360: $228-229$

Luft F, Gebert J, Schneider A, Melsheimer P, von Knebel Doeberitz M (1999) Frequent allelic imbalance of tumor suppressor gene loci in cervical dysplasia. Int J Gynecol Pathol 18: 374-380

Maciag PC, Schlecht NF, Souza PS, Franco EL, Villa LL, Petzl-Erler ML (2000) Major histocompatibility complex class II polymorphisms and risk of cervical cancer and human papillomavirus infection in Brazilian women. Cancer Epidemiol Biomarkers Prev 9: 1183-1191

Mohamed-Noor K, Quinn MA, Tan J (1997) Outcomes after cervical cold knife conization with complete and incomplete excision of abnormal epithelium: a review of 699 cases. Gynaecol Oncol 67: 34-38

Munoz N, Bosch FX, de Sanjose S, Herrero R, Castellsague X, Shah KV, Snijders PJ, Meijer CJ (2003) Epidemiologic classification of human papillomavirus types associated with cervical cancer. $N$ Engl J Med 348: $518-527$

Nagai Y, Maehama T, Asato T, Kanazawa K (2000) Persistence of human papillomavirus infection after therapeutic conization for CIN 3: is it an alarm for disease recurrence? Gynecol Oncol 79: 294-299

Pett MR, Alazawi WOF, Roberts I, Dowen S, Smith DI, Stanley MA, Coleman N (2004) Acquisition of high-level chromosomal instability is associated with integration of human papillomavirus type 16 in cervical keratinocytes. Cancer Res 64: 1359-1368

Plug-DeMaggio AW, Sundsvold T, Wurscher MA, Koop JI, Klingelhutz AJ, McDougall JK (2004) Telomere erosion and chromosomal instability in cells expressing the HPV oncogene 16E6. Oncogene 23: 3561-3571

Pulido HA, Fakruddin MJ, Chatterjee A, Esplin ED, Beleno N, Martinez G Posso H, Evans GA, Murty VV (2000) Identification of a 6-cM minimal deletion at 11q23.1-23.2 and exclusion of PPP2R1B gene as a deletion target in cervical cancer. Cancer Res 60: 6677-6682

Remmink AJ, Walboomers JM, Helmerhorst TJ, Voorhorst FJ, Rozendaal L Risse EK, Meijer CJ, Kenemans P (1995) The presence of persistent highrisk HPV genotypes in dysplastic cervical lesions is associated with progressive disease: natural history up to 36 months. Int J Cancer 61: $306-311$

Roberts I, Gordon A, Wang R, Pritchard-Jones K, Shipley J, Coleman N (2001) Molecular cytogenetic analysis consistently identifies translocations involving chromosomes 1,2 and 15 in five embryonal rhabdomyosarcoma cell lines and a PAX-FOXO1A fusion gene negative alveolar rhabdomyosarcoma cell line. Cytogenet Cell Genet 95: 134-142

Roberts I, Wienberg J, Nacheva E, Grace C, Griffin D, Coleman N (1999) Novel method for the production of multiple colour chromosome paints for use in karyotyping by fluorescence in situ hybridisation. Genes Chromosomes Cancer 25: $241-250$

Sanoudou D, Tingby O, Ferguson-Smith MA, Collins VP, Coleman N (2000) Analysis of pilocytic astrocytoma by comparative genomic hybridization. Br J Cancer 82: $1218-1222$

Sasieni PD (2000) Human papillomavirus screening and cervical cancer prevention. J Am Med Womens Assoc 55: 216-219

Stanley MA, Browne HM, Appleby M, Minson AC (1989) Properties of a non-tumorigenic human cervical keratinocyte cell line. Int J Cancer 43: $672-676$

Steenbergen RD, Walboomers JM, Meijer CJ, van der Raaij-Helmer EM, Parker JN, Chow LT, Broker TR, Snijders PJ (1996) Transition of human papillomavirus type 16 and 18 transfected human foreskin keratinocytes towards immortality: activation of telomerase and allele losses at 3p, 10p 11q and/or 18q. Oncogene 13: $1249-1257$

Steenbergen RDF, Kramer D, Meijer CJLM, Walboomers JMM, Trott DA, Cuthbert AP, Newbold RF, Overkamp WJI, Zdzienicka MZ, Snijders PJ (2001) Telomerase suppression by chromosome 6 in a human papillomavirus type 16-immortalized keratinocyte cell line and in a cervical cancer cell line. J Natl Cancer Inst 93: 865-872

Strauss S, Jordens JZ, McBride D, Sonnex C, Edwards S, Desselberger U, Watt P, Gray JJ (1999) Detection and typing of human papillomavirus DNA in paired urine and cervical scrapes. Eur J Epidemiol 15: $537-543$

Thorland EC, Myers SL, Persing DH, Sarkar G, McGovern RM, Gostout BS, Smith DI (2000) Human papillomavirus type 16 integrations in cervical tumors frequently occur in common fragile sites. Cancer Res 60: $5916-5921$

Tonon SA, Picconi MA, Bos PD, Zinovich JB, Galuppo J, Alonio LV, Teyssie AR (2001) Physical status of the E2 human papilloma virus 16 viral gene in cervical preneoplastic and neoplastic lesions. J Clin Virol 21: 129-134

Umayahara K, Numa F, Suehiro Y, Sakata A, Nawata S, Ogata H, Suminami Y, Sakamoto M, Sasaki K, Kato H (2002) Comparative genomic hybridization detects genetic alterations during early stages of cervical cancer progression. Genes Chromosomes Cancer 33: 98 - 102

Wentzensen N, Ridder R, Klaes R, Vinokurova S, Schaefer U, Doeberitz MK (2002) Characterization of viral-cellular fusion transcripts in a large series of HPV16 and 18 positive anogenital lesions. Oncogene 21: $419-426$

Zitzelsberger H, Lehmann L, Werner M, Bauchinger M (1997) Comparative genomic hybridisation for the analysis of chromosomal imbalances in solid tumours and haematological malignancies. Histochem Cell Biol 108: $403-417$ 